Received 00th January 20xx, Accepted 00th January 20xx DOI: $10.1039 / x 0 x \times 00000 x$

\section{Synergism of anisotropic and computational NMR methods reveals the likely configuration of phormidolide $A$}

\author{
Ikenna E. Ndukwe, ${ }^{a}$ Xiao Wang, ${ }^{b}$ Nelson Y. S. Lam, ${ }^{c}$ Kristaps Ermanis, ${ }^{c}$ Kelsey L. Alexander, ${ }^{d}$ \\ Matthew Bertin, ${ }^{\mathrm{e}}$ Gary E. Martin, ${ }^{\mathrm{f}}$ Garrett Muir, ${ }^{\mathrm{g}}$ lan Paterson, ${ }^{\mathrm{c}}$ Robert Britton, ${ }^{\mathrm{g}}$ Jonathan M.

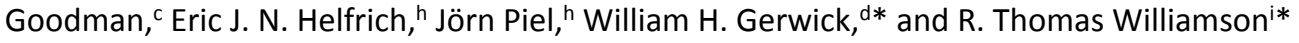

\begin{abstract}
Characterization of the complex molecular scaffold of the marine polyketide natural product phormidolide $A$ represents a challenge that has persisted for nearly two decades. In light of discordant results arising from recent synthetic and biosynthetic reports, a rigorous study of the configuration of phormidolide $A$ was necessary. This report outlines a synergistic effort employing computational and anisotropic NMR investigation, that provided orthogonal confirmation of the reassigned side chain, as well as supporting a further correction of the $\mathrm{C} 7$ stereocenter.
\end{abstract}

Phormidolide A (Fig. 1C) was isolated from the marine cyanobacterium Leptolyngbya sp. (strain ISB3NOV94-8A) and demonstrated mid-range toxicity in the brine shrimp model. ${ }^{1}$ Structurally, phormidolide A contains a 16-membered macrocycle linked to a pendant polyol side chain terminating in bromomethoxydiene; a signature motif shared with the related congeners phormidolide $\mathrm{B}, \mathrm{C}$ and oscillariolide (see $\mathrm{SI}$ )..$^{2,3}$ The planar structure of phormidolide $A$ was established from partial structures using ${ }^{1} \mathrm{H},{ }^{13} \mathrm{C}$, COSY, multiplicity-edited $\mathrm{HSQC}$, and HMBC NMR experiments. ${ }^{1}$ Additional confirmation for this assignment was provided by INADEQUATE and ACCORDADEQUATE data acquired on a ${ }^{13} \mathrm{C}$-enriched sample. ${ }^{1}$ The relative configuration of the tetrahydrofuran (THF) ring found in phormidolide $\mathrm{A}$ and the related congener oscillariolide was determined to be identical by conventional ROESY

a. Complex Carbohydrate Research Center, University of Georgia, Athens, Ga. USA.

b. Analytical Research \& Development, Merck \& Co. Inc. Rahway, NJ, USA

c. University Chemical Laboratory, Lensfield Road, Cambridge, CB2 1EW, UK.

d. Scripps Institution of Oceanography and Skaggs School of Pharmacy and

Pharmaceutical Sciences, University of California, San Diego, San Diego, CA, USA and Department of Chemistry, University of California, San Diego, San Diego, CA, USA.

e. Department of Chemistry, University of Rhode Island, Kingston, RI, USA.

f. Department of Chemistry, Seton Hall University, South Orange, NJ, USA.

g. Department of Chemistry, Simon Fraser University, 8888 University Drive, Burnaby, British Columbia, V5A 1S6, Canada

h. Institute of Microbiology, Eidgenössische Technische Hochschule (ETH) Zürich, Zurich, $\mathrm{CH}$.

Department of Chemistry \& Biochemistry, University of North Carolina Wilmington, Wilmington, NC USA.

† Footnotes relating to the title and/or authors should appear here.

Electronic Supplementary Information (ESI) available: [details of any supplementary information available should be included here]. See DOI: 10.1039/x0xx00000x

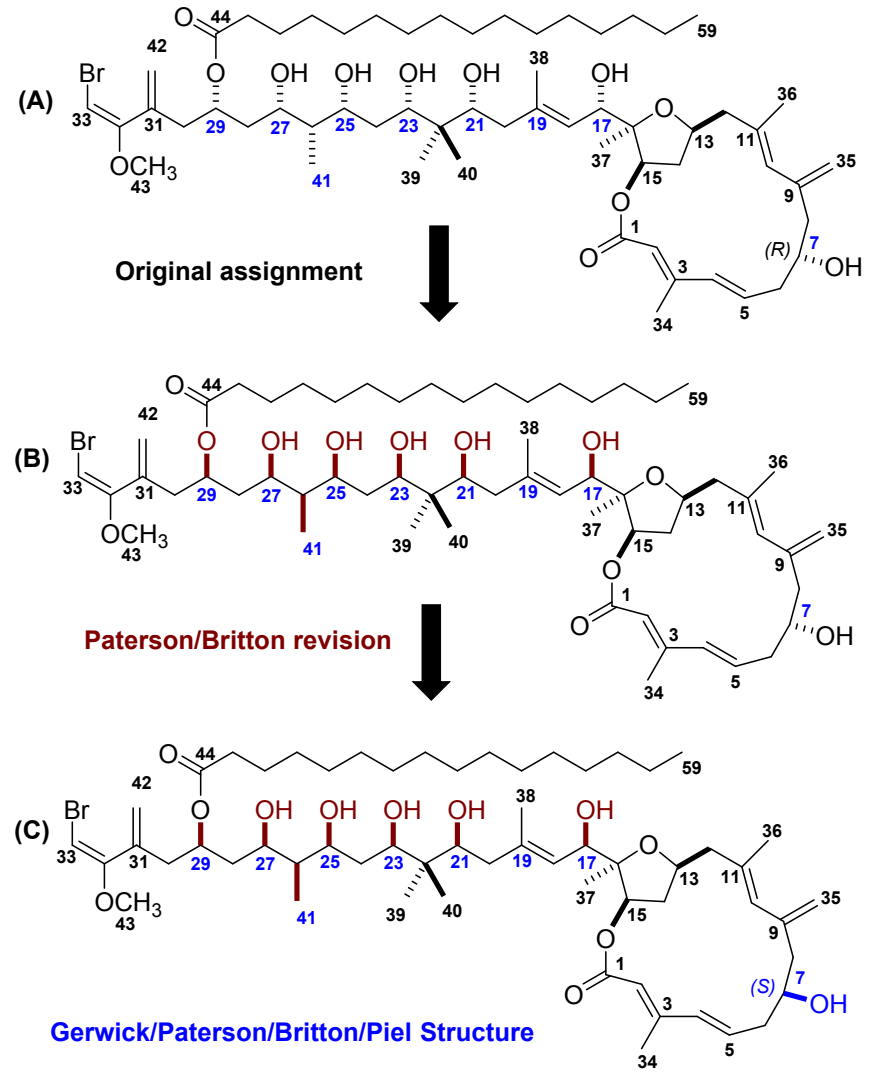

Figure 1: Schematic representation of phormidolide A stereochemical reassignment. (A) Previous incorrect structure, (B) synthetic revision of the side chain and (C) fully revised structure following computational and anisotropic NMR analysis disclosed in this work

experiments. ${ }^{1}$ Additional insight pertaining to the relative configuration for the remainder of the molecule was obtained by the then new J-based configuration analysis (JBCA) method. ${ }^{4-8}$ Since that time, this method has been shown to be extremely robust except in unusual cases, such as relating the configuration from the 2- or 6-position of a tetrahydropyran moiety to an adjacent oxygenated stereogenic center. ${ }^{9} \mathrm{By}$ 
analogy, the relative configuration from the $\mathrm{C} 17$ stereocenter to $\mathrm{C} 16$ of the neighboring tetrahydrofuran ring in phormidolide A was also shown to be problematic. In the case of phormidolide $\mathrm{A}$, the configuration at $\mathrm{C} 17$ was assigned as anti relative to $\mathrm{O} 16$ based on a semi-quantitative utilization of the magnitude of ${ }^{3} J_{\mathrm{CH}}$ correlations from $\mathrm{H} 17$ to $\mathrm{C} 15$ and to $\mathrm{CH}_{3}-37$, respectively. This anti arrangement between $\mathrm{H} 17$ and $\mathrm{O} 16$ was corroborated by ROESY correlations from the $\mathrm{CH}_{3}-37$ to $\mathrm{H} 18 .{ }^{1}$ In a recent report, synthetic work by the Paterson and Britton groups has demonstrated that the relative configuration at C17 should be assigned as syn relative to $\mathrm{O} 16$ (Fig 1B). ${ }^{10}$ Their work also showed that while the relative configuration between $\mathrm{C} 17$ and the remainder of the polyol chain (C21-C33) was assigned correctly in the original work, the reassignment at C17 inferred that the corresponding inversion of configuration at C21, C23, C25, C26, C27 and C29 was necessary.

Furthermore, in the original report, the absolute configuration at $C 7$ was assigned as $R$ through a set of variable temperature NMR experiments performed on a diacetonideprotected phormidolide. ${ }^{1}$ Since the relative configuration was established through extensive JBCA and NMR analyses, the absolute configuration of $\mathrm{C} 7$ was relayed to the THF ring, and to the remainder of the side chain to give the reported structure (Fig 1A). In 2016, the Gerwick group performed a complete biosynthetic gene cluster analysis on phormidolide that cast doubt on the $7 R$ (or $\mathrm{L}-\mathrm{OH} 7$ ) absolute configuration and therefore the stereochemistry of the natural product as a whole. ${ }^{11}$ To address this issue, the phormidolide A macrocycle was hydrolyzed and both the $R$ and $S$ a-methoxy- $\alpha$ trifluoromethylphenylacetic acid (MTPA) esters were synthesized from the triacetonide derivative, and subsequent spectroscopic analysis initially appeared to agree with the original assignment (Fig S6). This apparent conundrum with the biogenetic data was resolved after noting a prior report from the Reynolds group, ${ }^{12}$ which suggested that the anomalous L$\mathrm{OH}$ configuration (corresponding to the $7 R$ ) can indeed be catalytically generated from a type-B ketoreductase. Interestingly, a subsequent report from the Piel group on the genomic prediction and isolation of the leptolyngbyalides, a group of compounds closely related to the phormidolides, proposed not only the inversion of the configuration at $\mathrm{C7}$ for phormidolide $A$, but also the remainder of the natural product. ${ }^{13}$

Following the Piel report, a structural analysis of the ROESY, E.COSY, and HSQMBC data utilized for the initial assignment suggested that the configuration at $\mathrm{C} 7$ could be inverted without significantly altering the reported correlations for phormidolide A. Interestingly, the molecular mechanics (MM) structure presented in the original report, as well as more recent $\mathrm{MM}$ optimizations for both macrocyclic $\mathrm{C} 7$ epimers, provided structures that would allow the logical explanation of all scalar and dipolar coupling data (Fig S7).

At this point, three configurations for phormidolide $A$ have been proposed, namely the original assignment (Fig 1A), ${ }^{1}$ the Paterson/Britton assignment (Fig 1B $)^{10}$ and the Piel assignment ${ }^{13}$. In light of our ongoing interest in the total

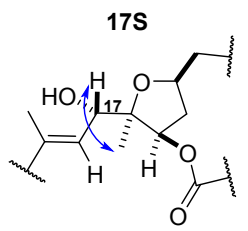

One conformation Anti $=3.3 \mathrm{~Hz}$
17R View Article Online

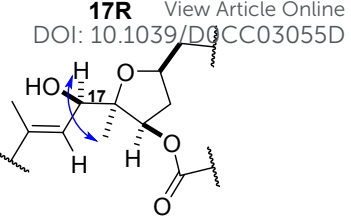

Two conformations $(\sim 1: 1)$

Anti $=3.9 \mathrm{~Hz}$

Gauche $=4.3 \mathrm{~Hz}$
Average $=4.1 \mathrm{~Hz}$

\section{Experimental $=4.1 \mathrm{~Hz}$}

Figure 2: C20-trucated phormidolide A C17-epimers ( $S$ and $R$ ) - complete macrolide not shown - that were utilized for computational investigation of the relative configuration of $\mathrm{C} 17$ with respect to the THF ring.

synthesis of phormidolide $A$ and the application of advanced NMR methods to structural/stereochemical assignment, we embarked on a multi-pronged approach focused on conclusively defining the configuration of phormidolide $A$, which is necessary to pin down an apparently dynamic target. Notably, we sought to leverage the advances in ab initio NMR methods, ${ }^{14}$ in conjunction with recently reported Residual Chemical Shift Anisotropy (RCSA) techniques, ${ }^{15-20}$ to independently verify the most likely structure for the natural product, as well as demonstrate the capability of these methods in deconvoluting a flexible and complex structure as challenging as phormidolide $A$.

Our first step towards verifying the stereochemical identity of phormidolide A was to investigate the apparent stereochemical mis-assignment at C17. DFT scalar coupling constant calculations of C17-epimers ( $R$ and $S)$ of the C20truncated sub-unit of phormidolide A (Fig 2) at GIAO-B3LYP2124/6-31G(d)//M06-2X25/6-31+G(d,p), uncovered a reversal of the conventional ${ }^{3} J_{\mathrm{CH}}$ values for anti vs gauche configuration in these systems. The $17 \mathrm{~S}$ configuration provided a single low energy conformation, stabilized by an $\mathrm{OH}-17$ to 016 gauche hydrogen bond. The DFT-derived anti $\mathrm{H} 17-\mathrm{CH}_{3}-37^{3} J_{\mathrm{CH}}$ coupling constant for this conformation was $3.3 \mathrm{~Hz}$. Conversely, the $17 R$ configuration provided two alternate conformations in a $\sim 1: 1$ ratio, with $\mathrm{OH}-17$ to $\mathrm{O} 15$ and $\mathrm{OH}-17$ to $\mathrm{O} 16$ gauche hydrogen bonds, respectively. Whereas the former is in an anti-parallel conformation for $\mathrm{H} 17-\mathrm{CH}_{3}-37$ (with a ${ }^{3} J_{\mathrm{CH}}$ coupling constant of $3.9 \mathrm{~Hz}$ ), the latter is in a gauche conformation (exhibiting a ${ }^{3} J_{\mathrm{CH}}$ of $4.3 \mathrm{~Hz}$ ). Reanalysis of the original spectroscopic data with modern data processing tools revealed a coupling of $4.1 \mathrm{~Hz}$, which is in seamless agreement with the averaged DFT prediction for the $17 R$ conformers (Fig 2). This anomalous result obfuscates the conclusive assignment of $\mathrm{C} 17$ through empirical JBCA, and thus requires more advanced analytical techniques to verify the configuration, as reported here.

Encouraged by the insight provided by $a b$ initio methods, and recalling the uncertainty surrounding the assignment of $7 R$ in the original report, DP4 calculations of the ${ }^{1} \mathrm{H}$ and ${ }^{13} \mathrm{C}$ chemical shifts of the two truncated macrocycle C7-epimers of phormidolide A (Fig S11) were undertaken. ${ }^{26}$ Geometries, shifts and conformer energies were calculated using DFT conditions found to be optimal in recent studies. ${ }^{27,28}$ The statistical model used was also tailored to the particular computational 


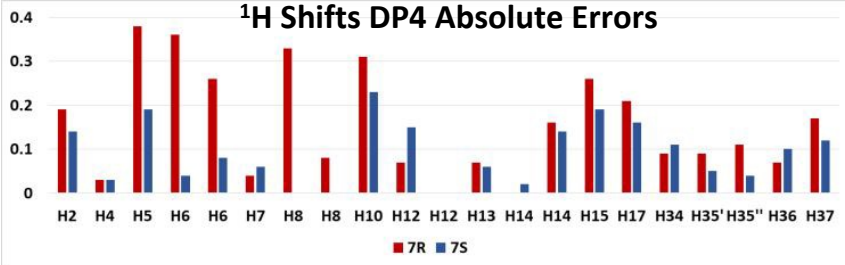

${ }^{13} \mathrm{C}$ Shifts DP4 Absolute Errors

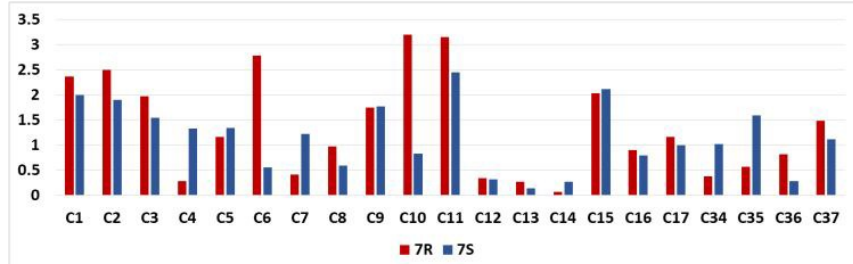

Figure 3: NMR shift DFT GIAO prediction errors (in absolute value mode) after internal scaling for ${ }^{1} \mathrm{H}$ (top) and ${ }^{13} \mathrm{C}$ (bottom). Geometries: B3LYP/6-31G**; shifts: mPW1PW91/6 311G*; conformer energies: M06-2X/def2-TZVP.

conditions. DP4 calculations suggested a $100 \%$ probability that the original assignment $(7 R)$ should be inverted, i.e. the $7 S$ configuration. This conclusion was further supported by the calculated chemical shifts, where initially large prediction errors were significantly reduced in the reassigned structure (Fig $\mathbf{3}$ ).

In addition, DFT calculations of ${ }^{3} J_{\mathrm{HH}},{ }^{2} \mathrm{~J}_{\mathrm{CH}}$, and ${ }^{3} \mathrm{~J}_{\mathrm{CH}}$ coupling constants (GIAO-B3LYP/6-31G(d)//M06-2X/6-31+G(d,p)) and ${ }^{13} \mathrm{C}$ NMR chemical shifts (GIAO-mPW1PW9129/6-311+G(2d,p) //M06-2X/6-31+G(d,p)), also indicated the 75 assignment (Fig 4). A recent report has demonstrated the utility of long-range $J$ coupling constants for diastereomeric analysis. ${ }^{30}$ Due to the computational demands of modelling such a highly flexible molecule, the models were truncated at C24 with only eight isomers considered. These isomers corresponded to isomeric permutations at the $\mathrm{C} 7, \mathrm{C} 17$ and $\mathrm{C} 21 / \mathrm{C} 23$ stereocenters. In this context, SRS would mean a $7 S, 17 R$ and $21 S$ configuration, respectively - with the THF fragment configuration remaining unchanged as shown in Fig $\mathbf{1 A}$ (n.b.: this notation also corresponds to $21 S / 23 R$, which are considered as a pair in each model and inverted simultaneously). The Mean Absolute Error (MAE) of experimental vs. DFT-derived scalar coupling and chemical shift values (up to $\mathrm{C} 21$ but excluding $\mathrm{sp}^{2}$ carbons due to errors associated with the DFT functional used $)^{31,32}$ not only favor the $7 S$ configuration but also the $17 R$ configuration (Fig $1 C$ ), with values of 0.84 and 1.22 , respectively (compared to 0.93 and 1.36 computed for the SSR configuration). Interestingly, including the $\mathrm{sp}^{2}$ carbon chemical shifts (previously excluded) in the statistical analysis also favor the $S R S$ configuration (Fig S13).

As a final check on the revised configuration of phormidolide A (Fig 1C), we further embarked on an orthogonal structure verification process - leveraging anisotropic NMR parameters to generate an unbiased orthogonal evaluation of structural accuracy. Anisotropic NMR data, including Residual Dipolar Coupling (RDC) and Residual Chemical Shift Anisotropy (RCSA), have developed into powerful parameters for verification of proposed molecular constitution, and thereby increase confidence in assigned structures. ${ }^{15,17,18,33-39}$ In particular, ${ }^{13} \mathrm{C}$

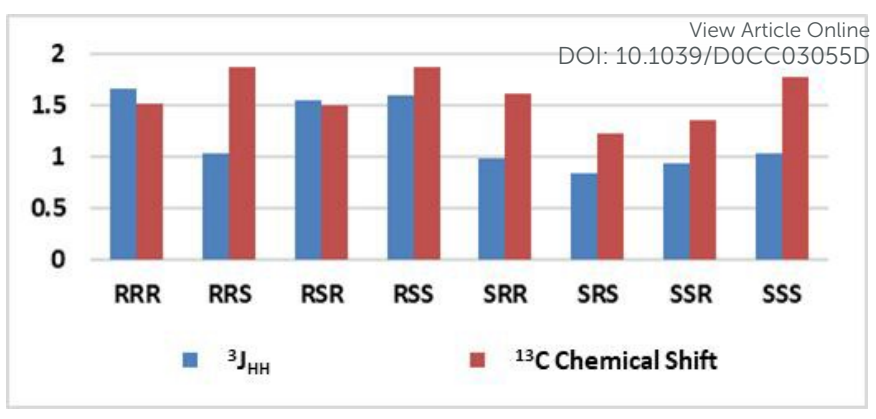

Figure 4: Bar charts of Mean Absolute Error (MAE) analysis of Expt. vs. DFT calculated J coupling constants (blue) and ${ }^{13} \mathrm{C}$ NMR chemical shift values (red). The MAE from both analyses favor the stereochemical revision of phormidolide $A$ to the structure shown in Fig $1 \mathrm{C}$, with the smallest values of 0.84 and 1.22 , respectively.

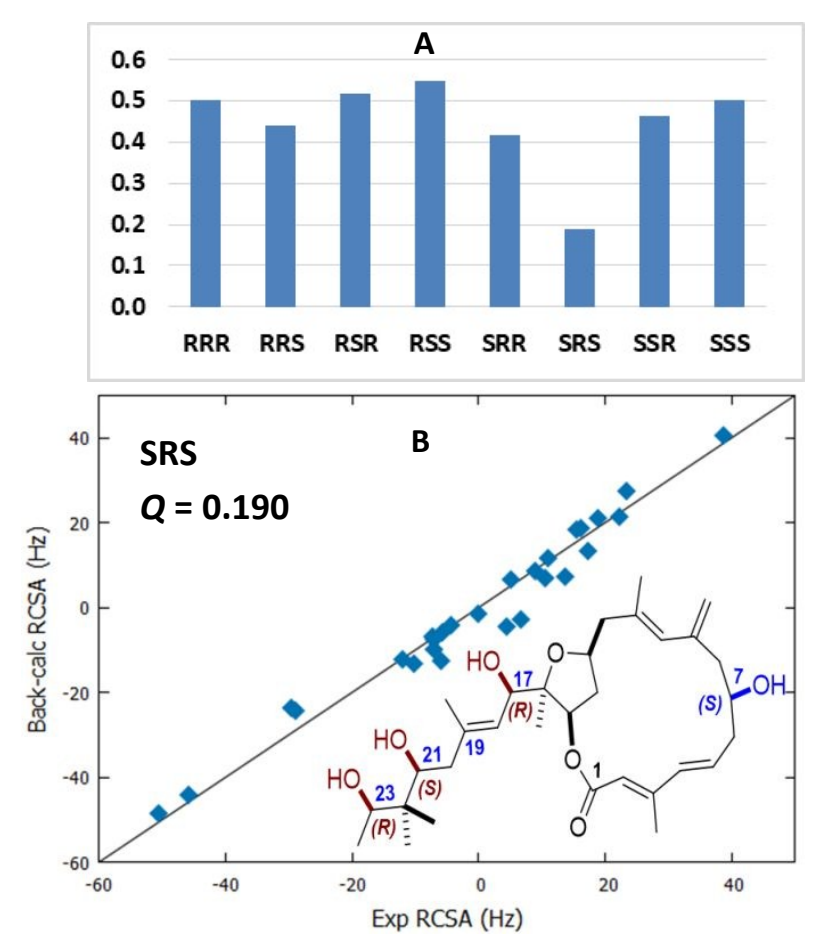

Figure 5: (A) Bar charts of $Q$-factors of isomeric phormidolide A structures, involving permutational stereo-inversion at C7, C17 and C21/C23 (highlighted inset, Fig. 5(B)), providing further evidence for the structural revision of phormidolide $A$ to SRS. (B) Correlation plot of experimental. RCSAs vs. theoretically calculated RCSAs of the revised structure of phormidolide $A(7 S, 17 R$ and $21 S / 23 R$, also termed $S R S$ for $C 7, C 17$ and C21 stereocenters), showing in the inset a $Q$-factor of 0.190 and the SRS C24-truncated phormidolide A fragment.

NMR chemical shift changes due to Residual Chemical Shift Anisotropy (RCSA) in appropriate liquid crystalline alignment media provide information on the relative orientations of nuclear chemical shielding tensors of the molecule that provides detailed information on molecular constitution and configuration that can be applied to molecules of even moderate flexibility. The eight diastereomeric phormidolide $A$ configurations (RRR, RRS, RSR, RSS, SSR, SRS, SSR and SSS), truncated at $\mathrm{C} 24$, were also used for anisotropic NMR evaluation. Due to the moderate flexibility of these diastereomers $(\mathrm{SI})$, which resulted in the generation of multiple 
conformations with $>1 \%$ Boltzmann populations, theoretically determined conformer electronic energies were used as additional constraints in the single-tensor singular value decomposition (SVD) analysis ${ }^{40}$ of the proposed structures. This approach potentially minimizes SVD overfitting of the comparatively sparse RCSA dataset. The superimposed conformations of the SRS configuration (Fig S14) demonstrates that single-tensor SVD analysis can be applied to these moderately flexible systems. To verify the Paterson/Piel revised side-chain configuration (and thus the revised stereochemistry of phormidolide A, Fig 1C, in general), RCSA data up to C22, including the diastereotopic methyl groups C39/C40, were also used for SVD analysis (summarized in Fig 5). Clearly, the SRS configuration agrees best with the measured RCSA data giving a $Q$-factor of 0.190 , while the nearest configuration $S R R$ gave a $Q$-factor of 0.418 . A revision of phormidolide A to the $S R S$ structure (i.e. $7 S, 17 R, 21 / 23 S$ in Fig $\mathbf{1 C}$ ) is thus warranted.

In this work, we report the synergistic application of $a b$ initio NMR prediction methods in combination with anisotropic NMR parameters to establish the likely structure of phormidolide $A$. Through the confluence of three advanced techniques previously unavailable, we highlight that the reassigned relative configuration between the THF core and the side chain was correctly formulated by Paterson/Britton, while independently confirming the stereochemical reassignment of $7 R$ to the epimeric $7 S$ configuration as proposed by Piel, but correcting the relative configuration to the THF ring. Notably, these results resolve the stereochemical discrepancies extant between the original isolation report, the Paterson/Britton and subsequent Piel reassignments with the biogenetic data presented by the Gerwick group. This work serves to demonstrate that without these analytical advancements over the last two decades, a complex structure such as phormidolide A would have been difficult to define. Work on the total synthesis of phormidolide $A$ is on-going, which will provide a definitive proof-of-structure as a final verification of the stereochemical reassignments proposed for this fascinating yet problematic natural product.

\section{Acknowledgements}

IEN acknowledges Parastoo Azadi and Christian Heiss from the University of Georgia for NMR spectroscopy (grant DESC0015662 to PA). KLA acknowledges Sebastian Rohrer from University of California, San Diego, for wet chemistry assistance, and financial support from NIH T32 GM067550. WHG acknowledges NIH GM107550. NYSL acknowledges the Woolf Fisher Trust for financial support. KE thanks Leverhulme Trust and Isaac Newton Trust for financial support.

\section{There are no conflicts to declare.}

\section{Notes and references}

1 R. T. Williamson, A. Boulanger, A. Vulpanovici, M. A. Roberts and W. H. Gerwick, J. Org. Chem., 2002, 67, 7927-7936.

2 A. Lorente, A. Gil, R. Fernández, C. Cuevas, F. Albericio and M. Álvarez, Chem. - A Eur. J., 2015, 21, 150-156.

3 M. Murakami, H. Matsuda, K. Makabe and K. Yamaguchi, Tetrahedron
Lett., 1991, 32, 2391-2394.

R. T. Williamson, B. L. Marquez, A. C. Barrios Sosa and Eiew Article Online Magn. Reson. Chem., 2003, 41, 379-385.

P. Cimino, G. Bifulco, A. Evidente, M. Abouzeid, R. Riccio and L. GomezPaloma, Org. Lett., 2002, 4, 2779-2782.

N. Matsumori, D. Kaneno, M. Murata, H. Nakamura and K. Tachibana, J. Org. Chem., 1999, 64, 866-876.

T. F. Molinski and B. I. Morinaka, Tetrahedron, 2012, 68, 9307-9343. G. Bifulco, P. Dambruoso, L. Gomez-Paloma and R. Riccio, Chem. Rev., 2007, 107, 3744-3779.

Y. Wakamiya, M. Ebine, N. Matsumori and T. Oishi, J. Am. Chem. Soc., 2020, 142, 3472-3478.

N. Y. S. Lam, G. Muir, V. R. Challa, R. Britton and I. Paterson, Chem. Commun., 2019, 55, 9717-9720.

M. J. Bertin, A. Vulpanovici, E. A. Monroe, A. Korobeynikov, D. H. Sherman, L. Gerwick and W. H. Gerwick, ChemBioChem, 2016, 17, 164173.

S. A. Bonnett, J. R. Whicher, K. Papireddy, G. Florova, J. L. Smith and K. A. Reynolds, Chem. Biol., 2013, 20, 772-783.

E. J. N. Helfrich, R. Ueoka, A. Dolev, M. Rust, R. A. Meoded, A. Bhushan, G. Califano, R. Costa, M. Gugger, C. Steinbeck, P. Moreno and J. Piel, Nat. Chem. Biol., 2019, 15, 813-821.

G. Lauro and G. Bifulco, European J. Org. Chem., 2020, 1-14.

Y. Liu, J. Saurí, E. Mevers, M. W. Peczuh, H. Hiemstra, J. Clardy, G. E. Martin and R. T. Williamson, Science 2017, 356, 5349.

Y. Liu, R. D. Cohen, K. R. Gustafson, G. E. Martin and R. T. Williamson, Chem. Commun., 2018, 54, 4254-4257.

I. E. Ndukwe, A. Brunskill, D. R. Gauthier, Y. L. Zhong, G. E. Martin, R. T. Williamson, M. Reibarkh and Y. Liu, Org. Lett., 2019, 21, 4072-4076. I. E. Ndukwe, X. Wang, I. Pelczer, M. Reibarkh, R. T. Williamson, Y. Liu and G. E. Martin, Chem. Commun., 2019, 55, 4327-4330.

Y. Liu, A. Navarro-Vázquez, R. R. Gil, C. Griesinger, G. E. Martin and R. T. Williamson, Nat. Protoc., 2019, 14, 217-247.

N. Nath, M. Schmidt, R. R. Gil, R. T. Williamson, G. E. Martin, A. Navarro-Vázquez, C. Griesinger and Y. Liu, J. Am. Chem. Soc., 2016, 138, 9548-9556.

P. J. Stephens, F. J. Devlin, C. F. Chabalowski and M. J. Frisch, J. Phys. Chem., 1994, 98, 11623-11627.

S. H. Vosko, L. Wilk and M. Nusair, Can. J. Phys., 1980, 58, 1200-1211. C. Lee, W. Yang and R. G. Parr, Phys. Rev. B, 1988, 37, 785-789. A. D. Becke, J. Chem. Phys., 1993, 98, 5648-5652.

Y. Zhao and D. G. Truhlar, Theor. Chem. Acc., 2008, 120, 215-241. S. G. Smith and J. M. Goodman, J. Am. Chem. Soc., 2010, 132, 1294612959.

K. Ermanis, K. E. B. Parkes, T. Agback and J. M. Goodman, Org. Biomol. Chem., 2019, 17, 5886-5890.

A. Howarth, K. Ermanis and J. M. Goodman, Chem. Sci., 2020. C. Adamo and V. Barone, J. Chem. Phys., 1998, 108, 664-675. M. R. M. Koos, A. Navarro-Vázquez, C. Anklin and R. R. Gil, Angew. Chemie, 2020, 132, 3966-3969.

M. W. Lodewyk, M. R. Siebert and D. J. Tantillo, Chem. Rev., 2012, 112, 1839-1862.

A. M. Sarotti and S. C. Pellegrinet, J. Org. Chem., 2009, 74, 7254-7260. A. Ibáñez de Opakua, F. Klama, I. E. Ndukwe, G. E. Martin, R. T. Williamson and M. Zweckstetter, Angew. Chemie Int. Ed., 2020, 1-6. D. J. Milanowski, N. Oku, L. K. Cartner, H. R. Bokesch, R. T. Williamson, J. Saurí, Y. Liu, K. A. Blinov, Y. Ding, X.-C. Li, D. Ferreira, L. A. Walker, S. Khan, M. T. Davies-Coleman, J. A. Kelley, J. B. McMahon, G. E. Martin and K. R. Gustafson, Chem. Sci., 2018, 9, 307-314.

E. Mevers, J. Saurí, Y. Liu, A. Moser, T. R. Ramadhar, M. Varlan, R. T. Williamson, G. E. Martin and J. Clardy, J. Am. Chem. Soc., 2016, 138, 12324-12327.

M. Schwab, V. Schmidts and C. M. Thiele, Chem. - A Eur. J., 2018, 24, 14373-14377.

C. M. Thiele, European J. Org. Chem., 2008, 2008, 5673-5685. C. M. de Melo Sousa, R. B. Giordani, W. A. M. de Almeida, C.

Griesinger, R. R. Gil, A. Navarro-Vázquez and F. Hallwass, Magn. Reson. Chem., 2019, 1-8.

A. Navarro-Vázquez, R. R. Gil and K. Blinov, J. Nat. Prod., 2018, 81, 203-210.

J. A. Losonczi, M. Andrec, M. W. Fischer and J. H. Prestegard, J. Magn. Reson., 1999, 138, 334-342. 